\title{
Lack of $\alpha 8$ integrin leads to morphological changes in renal mesangial cells, but not in vascular smooth muscle cells
}

\author{
Ines Marek ${ }^{1 \dagger}$, Gudrun Volkert ${ }^{2 \dagger}$, Angelika Jahn ${ }^{1}$, Fabian Fahlbusch ${ }^{1}$, Christina Zürn², Zehra Özcan², \\ Margarete Goppelt-Struebe ${ }^{2}$, Karl F Hilgers ${ }^{2}$, Wolfgang Rascher ${ }^{1}$, Andrea Hartner ${ }^{1 *}$
}

\begin{abstract}
Background: Extracellular matrix receptors of the integrin family are known to regulate cell adhesion, shape and functions. The $\alpha 8$ integrin chain is expressed in glomerular mesangial cells and in vascular smooth muscle cells. Mice deficient for $\alpha 8$ integrin have structural alterations in glomeruli but not in renal arteries. For this reason we hypothesized that mesangial cells and vascular smooth muscle cells differ in their respective capacity to compensate for the lack of $\alpha 8$ integrin.
\end{abstract}

Results: Wild type and $\alpha 8$ integrin-deficient mesangial cells varied markedly in cell morphology and expression or localization of cytoskeletal molecules. In $\alpha 8$ integrin-deficient mesangial cells $\alpha$-smooth muscle actin and CTGF were downregulated. In contrast, there were no comparable differences between $\alpha 8$ integrin-deficient and wild type vascular smooth muscle cells. Expression patterns of integrins were altered in $\alpha 8$ integrin-deficient mesangial cells compared to wild type mesangial cells, displaying a prominent overexpression of $\alpha 2$ and $\alpha 6$ integrins, while expression patterns of the these integrins were not different between wild type and $\alpha 8$ integrin-deficient vascular smooth muscle cells, respectively. Cell proliferation was augmented in $\alpha 8$ integrin-deficient mesangial cells, but not in vascular smooth muscle cells, compared to wild type cells.

Conclusions: Our findings suggest that $\alpha 8$ integrin deficiency has differential effects in mesangial cells and vascular smooth muscle cells. While the phenotype of vascular smooth muscle cells lacking $\alpha 8$ integrin is not altered, mesangial cells lacking $\alpha 8$ integrin differ considerably from wild type mesangial cells which might be a consequence of compensatory changes in the expression patterns of other integrins. This could result in glomerular changes in $\alpha 8$ integrin-deficient mice, while the vasculature is not affected in these mice.

\section{Background}

Integrin family receptors mediate cell-cell or cell-matrix interactions. Integrins are heterodimers consisting of an $\alpha$ and a $\beta$ subunit. At least $18 \alpha$ and $8 \beta$ chains are known to date, which combine to 24 integrin receptors [1]. Most receptors recognize more than one ligand and each ligand is capable of binding several integrins, which leads to a wide variety of possible interactions [2]. Many $\beta 1$ and $\beta 3$ integrins are receptors for extracellular matrix molecules mediating not only adhesion of cells

\footnotetext{
* Correspondence: andrea.hartner@uk-erlangen.de

† Contributed equally

${ }^{1}$ Hospital for Children and Adolescents, Universität Erlangen-Nürnberg,

Loschgestrasse 15, 91054 Erlangen, Germany

Full list of author information is available at the end of the article
}

but also conveying signals which affect cytoskeletal architecture and thus cell morphology and differentiation (reviewed in [3-5]): In renal cells, signaling via integrins can alter the expression of cytoskeletal proteins [6] and the arrangement of cytoskeletal components, which is mediated via integrin linked kinase $[7,8]$. Several studies have suggested a regulatory role for integrins in the differentiation of epithelial cells [9], podocytes [6], mesangial cells [10] or fibroblasts [11]. Moreover, integrin signaling is involved in epithelial to mesenchymal transition, a phenomenon frequently seen in models of renal fibrotic disease [12,13].

The $\alpha 8$ integrin chain is expressed predominantly on mesenchymal cells, like vascular smooth muscle cells, certain fibroblast cells and glomerular mesangial cells,

\section{Biomed Central}


where it serves as a receptor for fibronectin, vitronectin, tenascin-C fragments, osteopontin and nephronectin, but not for collagens [14-17]. Moreover, a role for $\alpha 8 \beta 1$ integrin in migration, proliferation and survival of cells was described $[18,19]$.

A few studies suggested that $\alpha 8$ integrin may be involved in cell differentiation processes: $\alpha 8$ integrin expression was reported to contribute to the maintenance of the smooth muscle cell differentiated phenotype, because downregulation of $\alpha 8$ integrin led to a severe reduction of $\alpha$-smooth muscle actin expression and an increase in cell motility [20], while overexpression of $\alpha 8$ integrin had the opposite effects [21]. In fibroblasts, the downregulation of $\alpha 8$ integrin resulted in epithelialization, possibly due to induced WT-1 expression [22].

A role for a8 integrin was suggested in atherosclerotic [23] and fibrotic diseases $[24,25]$. Changes in the cytoskeletal architecture of cells could influence their ability to adhere and migrate. This might have an important impact on the progression of atherosclerotic or fibrotic diseases. On the other hand, we did not observe alterations of the smooth muscle cell layers of renal arteries and arterioles in $\alpha 8$ integrin-deficient mice, whereas the glomerular mesangium of these mice was clearly abnormal [26]. Thus, we hypothesized that mesangial cells and vascular smooth muscle cells might be differently affected by a lack of $\alpha 8$ integrin. We investigated if the properties of mesangial and vascular smooth muscle cells isolated from $\alpha 8$ integrin-deficient mice differ from their respective counterparts cultured from wild type controls.

\section{Results}

Cultivated wild type and $\alpha 8$ integrin-deficient mouse mesangial cells (MCs) were tested for mRNA and protein expression of $\alpha 8$ integrin to confirm lack of $\alpha 8$ integrin expression in $\alpha 8$ integrin-deficient MCs and presence of $\alpha 8$ integrin in wild type cells. $\alpha 8$ integrin mRNA expression was readily detected in wild type MCs by real-time RT-PCR (Figure 1A), while $\alpha 8$ integrin mRNA expression was within background detection in $\alpha 8$ integrin-deficient MCs (Figure 1A). a8 integrin protein was detected in wild type MCs, but not in a8 integrin-deficient MCs by Western blot analysis (Figure 1B).

Wild type MCs in culture grew in a typical mesenchymal pattern (Figure 2A), while the morphology of $\alpha 8$ integrin-deficient MCs was different with a more compact cell shape lacking long protrusions. Moreover, $\alpha 8$ integrin-deficient MCs were arranged in groups forming cell islets (Figure 2B). F-actin fibers were frequently arranged parallel to the cytoplasma membrane in $\alpha 8$ integrin-deficient MCs (Figure 2D, F and 2H), while in wild type MCs f-actin fibers were spanning the whole cell

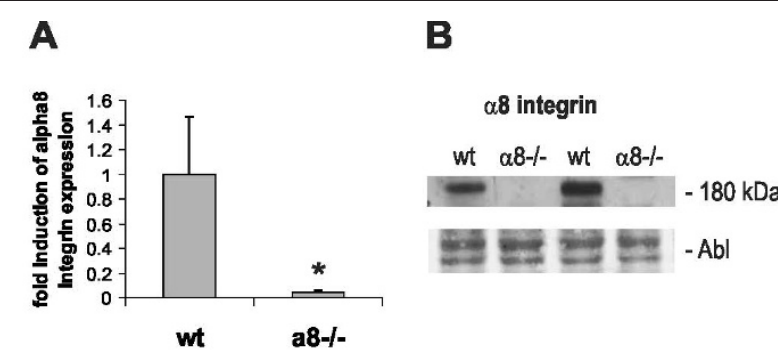

Figure 1 Mesangial cell expression of $\alpha 8$ integrin in wild type (wt) and $\alpha 8$ integrin-deficient mesangial cells ( $\alpha 8-/-)$. A: Realtime RT-PCR analysis of $\alpha 8$ integrin expression in wt and $\alpha 8_{-} /-$ mesangial cells. B: Western blot analysis of $\alpha 8$ integrin protein in wt and $\alpha 8$-/- mesangial cells. Amido black (Abl) staining of the blot served as loading control. Results are representative for at least 3 similar experiments. Data are means $\pm \mathrm{sd}$. ${ }^{*} \mathrm{p}<0.05$ vs. wt.

more irregularily (Figure 2C, E and 2G) after an attachment time of 24 hours. Focal contacts were shorter in $\alpha 8$ integrin-deficient MCs compared to wild type MCs and frequently arranged in bundles (Figure $2 \mathrm{D}$ and $2 \mathrm{~K}$ ). mRNA expression of $\alpha$-smooth muscle actin was clearly detectable in wild type MCs but downregulated in $\alpha 8$ integrin-deficient MCs almost to background levels (Figure 3A), $\alpha$-smooth muscle actin protein was barely detectable by Western blot analysis in $\alpha 8$ integrindeficient MCs (Figure 3B). While wild type MCs $\alpha$-smooth muscle actin stain was arranged in typical stress fibers, most $\alpha 8$ integrin-deficient MCs stained negative for $\alpha$-smooth muscle actin, except for some occasional staining in short cortical fibers along the plasma membrane (Figure 3C). Double staining for $\alpha$-smooth muscle actin and f-actin confirmed that in wild type MCs $\alpha$-smooth muscle actin is a component of stress fibers (Figure 3D). In $\alpha 8$ integrin-deficient MCs $\mathrm{f}$-actin staining is preserved despite the lack of $\alpha$-smooth muscle actin immunoreactivity, arguing for a contribution of other types of actin to f-actin-positive fibers (Figure 3D).

In contrast to MCs, vascular smooth muscle cells (VSMCs) downregulate $\alpha 8$ integrin expression after cell passaging in culture. In freshly isolated VSMCs of passage $1, \alpha 8$ integrin expression was readily detectable by real-time RT-PCR, while in VSMCs after 10 passages, $\alpha 8$ integrin became nearly undetectable (Figure 4A). For this reason, we only used freshly isolated VSMCs in passage 1 for our further experiments. Lack of $\alpha 8$ integrin expression in $\alpha 8$ integrin-deficient VSMCs was confirmed by real-time RT-PCR (Figure 4B) and Western blot analysis (Figure 4C).

In contrast to our findings in MCs, cell morphology was not different in wild type and $\alpha 8$ integrin-deficient VSMCs (Figure 5A and 5B). Moreover, the distribution 


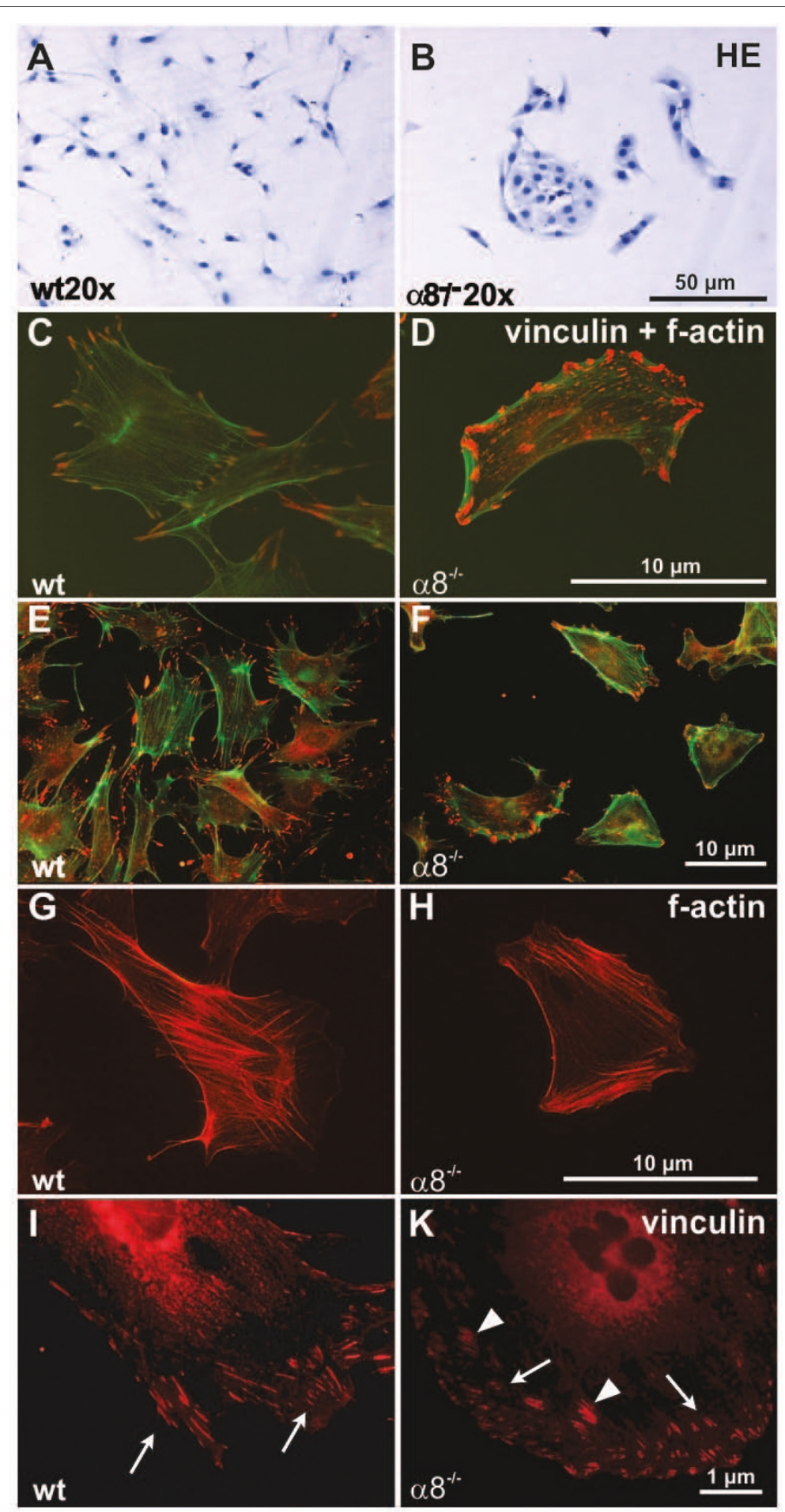

Figure 2 Comparison of wild type (wt) and $\alpha 8$ integrin-deficient ( $\alpha 8-/-)$ mesangial cell morphology after hematoxylin stain (A+B), immunofluorescent double staining for $f$-actin in green and vinculin in red (high magnification $C+D$, low magnification $E+F)$,

immunofluorescent staining for $\mathbf{f}$-actin alone $(\mathrm{G}+\mathrm{H})$ or immunofluorescent staining for vinculin alone (I+K). White arrows indicate focal contacts of the cells and white arrowheads indicate bundles of focal contacts in $\alpha 8-/$ - mesangial cells. 


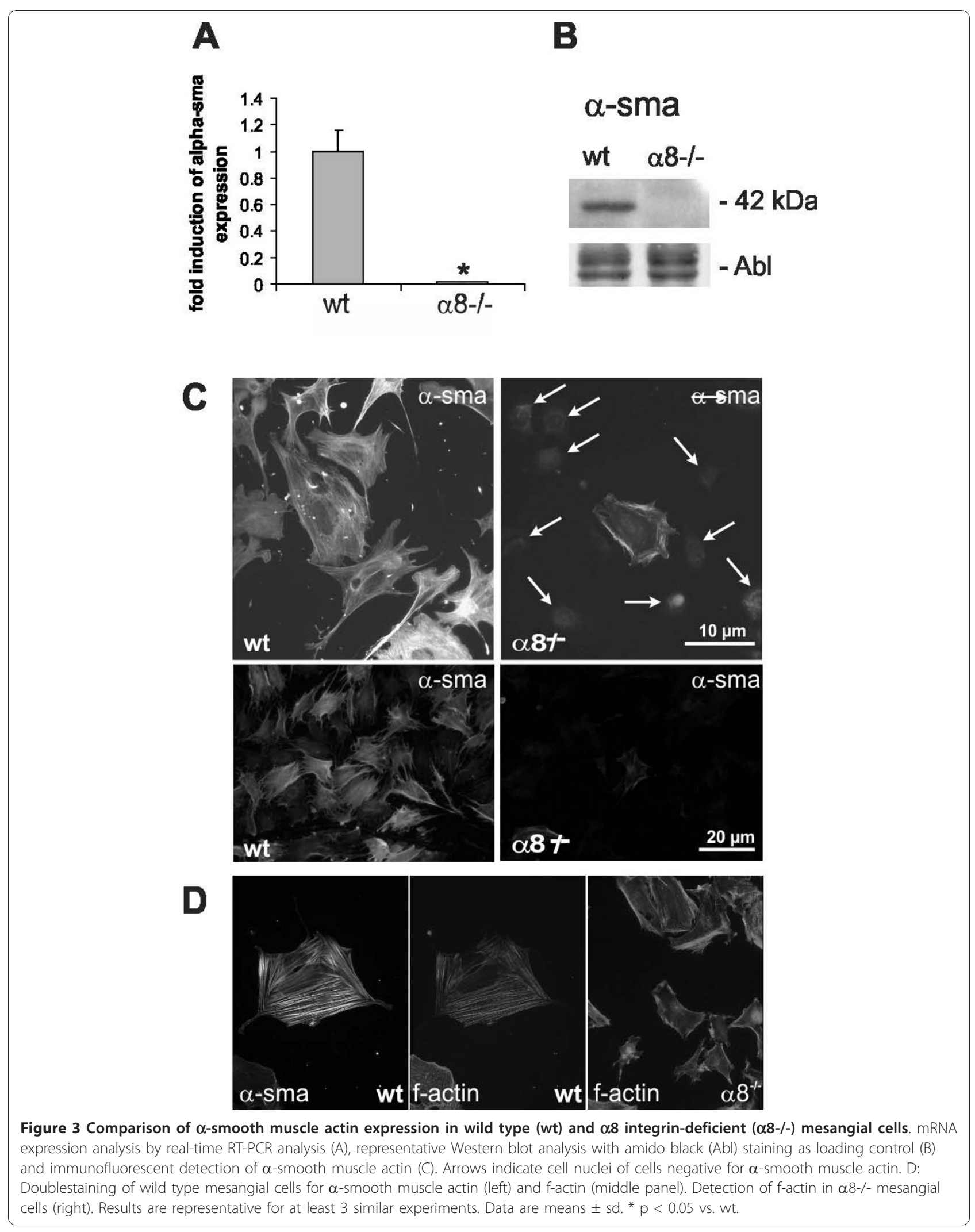



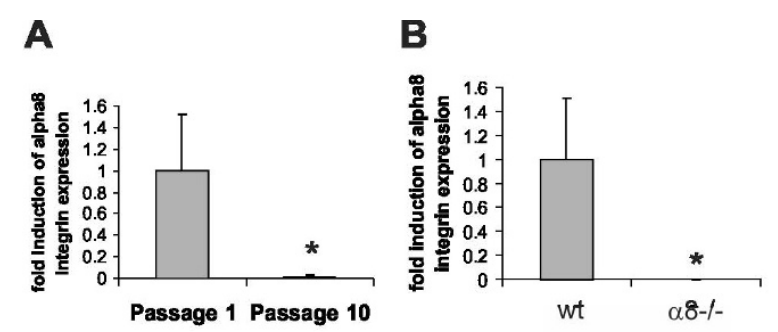

C

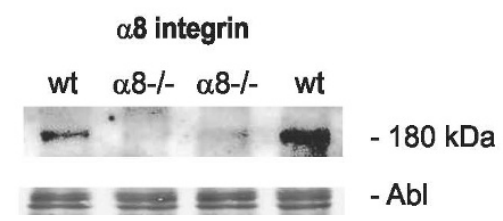

Figure 4 Expression of $\alpha 8$ integrin in vascular smooth muscle cells. Real-time RT-PCR analysis of $\alpha 8$ integrin expression in wild type (wt) vascular smooth muscle cells cultivated in passage 1 or 10 (A). Real-time RT-PCR analysis of $\alpha 8$ integrin expression in freshly isolated wt and $\alpha 8-/-$ vascular smooth muscle cells (B). Western blot analysis of $\alpha 8$ integrin expression in freshly isolated wt and $\alpha 8-/-$ vascular smooth muscle cells (C). Amido black (Abl) staining of the blot served as loading control. Results are representative for at least 3 similar experiments. Data are means $\pm \mathrm{sd}$. ${ }^{*} p<0.05$ vs. freshly isolated cells or wt, respectively.

of $\mathrm{f}$-actin fibers was not different in $\alpha 8$ integrin-deficient VSMCs compared to wild type VSMCs (Figure 5C and 5D) after 24 hours of attachment. Adherent $\alpha 8$ integrindeficient VSMCs developed focal contacts, which were comparable to the focal contacts of wild type VSMCs (Figure 5E and 5F).

Evaluation of $\alpha$-smooth muscle actin expression revealed no significant differences between wild type and $\alpha 8$ integrin-deficient VSMCs (Figure 6A). Western blot analysis revealed that $\alpha$-smooth muscle actin protein was abundant in wild type as well as $\alpha 8$ integrindeficient VSMCs (Figure 6B). $\alpha$-smooth muscle actin fibers were spanning the whole cell and were not reduced in $\alpha 8$ integrin-deficient VSMCs compared to wild types (Figure 6C).

As reorganisation of the actin cytoskeleton can lead to changes in the expression of connective tissue growth factor (CTGF), we assessed protein expression levels of CTGF in wild type and $\alpha 8$ integrin-deficient MCs in comparison to VSMCs. In MCs, a lack of $\alpha 8$ integrin resulted in downregulation of the protein expression of CTGF (Figure 7), while in $\alpha 8$ integrin-deficient VSMCs CTGF expression was not reduced (Figure 7).

We hypothesized that the differences in the effects of the lack of $\alpha 8$ integrin on cytoskeletal organization observed in MCs and VSMCs could be a consequence of a different regulation of other integrins in both cell types. Therefore, we compared expression patterns of several integrins in $\alpha 8$ integrin-deficient cells and wild type cells.
In $\alpha 8$ integrin-deficient MCs, induction of integrin chains $\alpha 1$, and even more prominently of $\alpha 2$ and $\alpha 6$, was detected when compared to wild type MCs (Figure 8A). In contrast, none of the investigated integrin chains was induced in $\alpha 8$ integrin-deficient VSMCs (Figure 8B).

We then hypothesized that MCs lacking $\alpha 8$ integrin might downregulate not only $\alpha$-smooth muscle actin but also additional mesenchymal markers. To analyze expression patterns of other mesenchymal markers, we performed real-time RT-PCR for vimentin and desmin and for the epithelial marker E-cadherin, because $\alpha 6$ integrins are known to upregulate E-cadherin-mediated adhesion [27]. In $\alpha 8$ integrin-deficient MCs the expression of desmin was significantly lower than in wild type MCs (Figure 9A), while in $\alpha 8$ integrin-deficient VSMCs desmin expression was not significantly different from desmin expression in wild type VSMCs (Figure 9B). On the other hand, vimentin expression was not affected by the lack of $\alpha 8$ integrin, in none of the cell types (Figure 9C and 9D). E-cadherin expression was barely above detection level in both wild type and $\alpha 8$ integrindeficient MCs, compared to its expression in liver cells used as positive control (Figure 9E). In VSMCs, expression of E-cadherin was not different in wild type and $\alpha 8$ integrin-deficient cells (Figure 9F).

To clarify if these differences of the properties of $\alpha 8$ integrin-deficient MCs and VSMCs have functional consequences, we performed proliferation assays. Our results show that $\alpha 8$ integrin-deficient MCs and VSMCs differ in their growth response to fetal calf serum when grown on fibronectin, a ligand for $\alpha 8$ integrin (Figure 10). While stimulation of $\alpha 8$ integrin-deficient MCs leads to significantly more proliferation than stimulation of wild type MCs, proliferation of wild type and $\alpha 8$ integrin-deficient VSMCs was not different.

\section{Discussion}

Taken together, a8 integrin-deficient MCs differed from wild type MCs with regard to morphology, cytoskeletal architecture and proliferative capacity, while a8 integrindeficient VSMCs did not differ from wild type VSMCs. This is in keeping with our previous in vivo findings suggesting changes in the glomerular mesangium but not in the media of renal arteries in a8 integrin-deficient mice [26], although in normal mice both structures contain mesenchymal cells expressing a8 integrin. a8 integrindeficient MCs downregulated expression of a-smooth muscle actin and desmin, but not vimentin, while expression of these mesenchymal proteins was not altered in a8 integrin-deficient VSMCs. a8 integrin-deficient MCs had higher expression levels of integrin chains a1, a 2 and a6 compared to wild type MCs. Similar differences were not detected between a8 integrin-deficient and wild type VSMCs. Moreover, increased proliferation rates due to a 

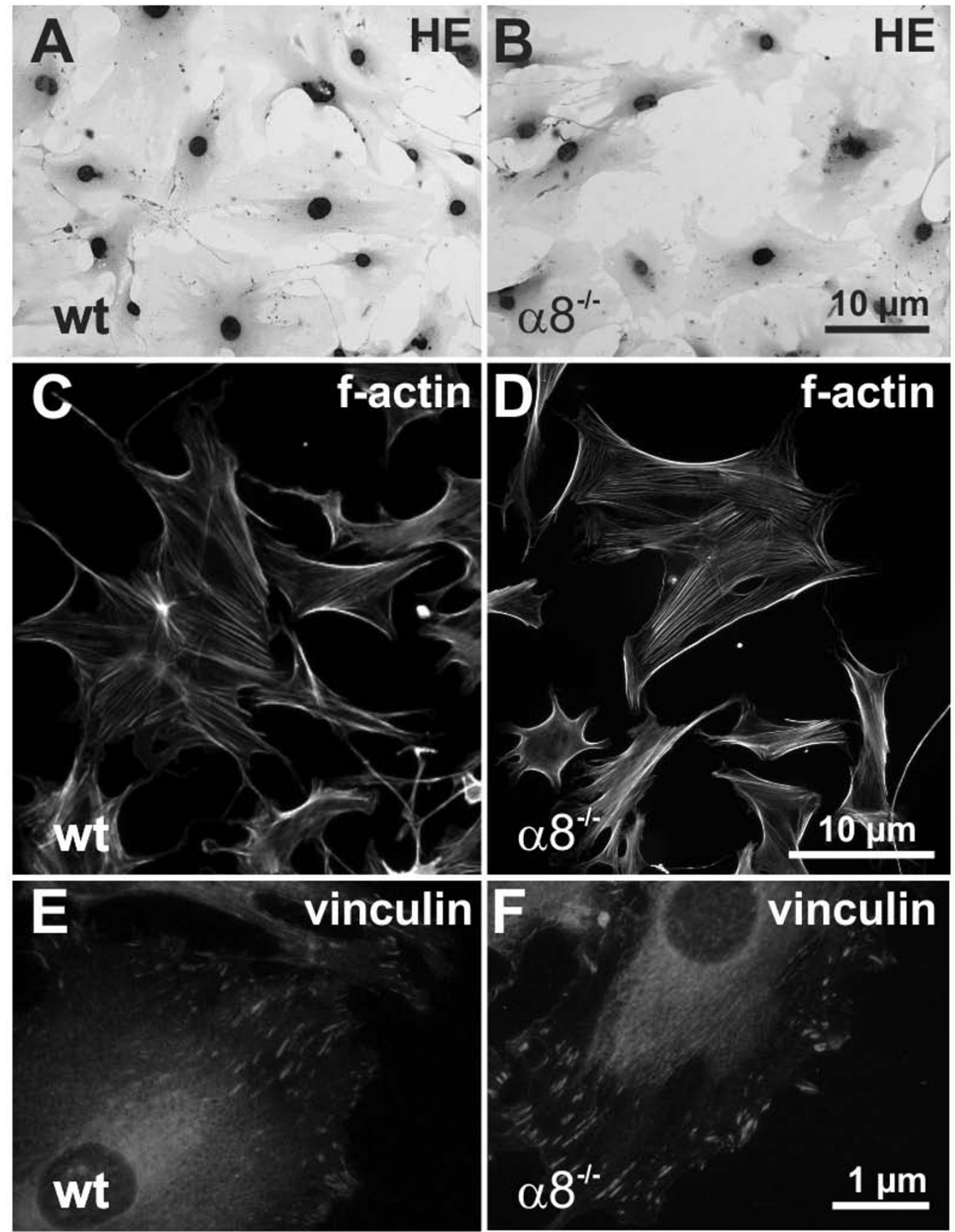

Figure 5 Comparison of wild type (wt) and $\alpha 8$ integrin-deficient ( $\alpha 8-/-)$ vascular smooth muscle cell morphology after hematoxylin stain $(A+B)$ or immunofluorescent staining for $f$-actin $(C+D)$ or immunofluorescent staining for vinculin $(E+F)$.

lack of a8 integrin were only detected in MCs, not in VSMCs.

Several studies show that integrins can contribute to cell differentiation and to the maintenance of the phenotype of the cell via outside-in signaling from the surrounding matrix to the cytoskeleton and small adapter molecules inside the cell $[4,28]$. Many integrins use a signaling pathway involving the $\beta 1$ integrin chain and integrin linked kinase to regulate the cytoskeletal architecture of the cell [29]. Moreover, integrins can alter the organization of the actin cytoskeleton via proteins of the rho family, which also regulate CTGF [30]. CTGF, besides having profibrotic function, can act as a mediator of growth arrest [31]. In MCs, disassembly of actin 

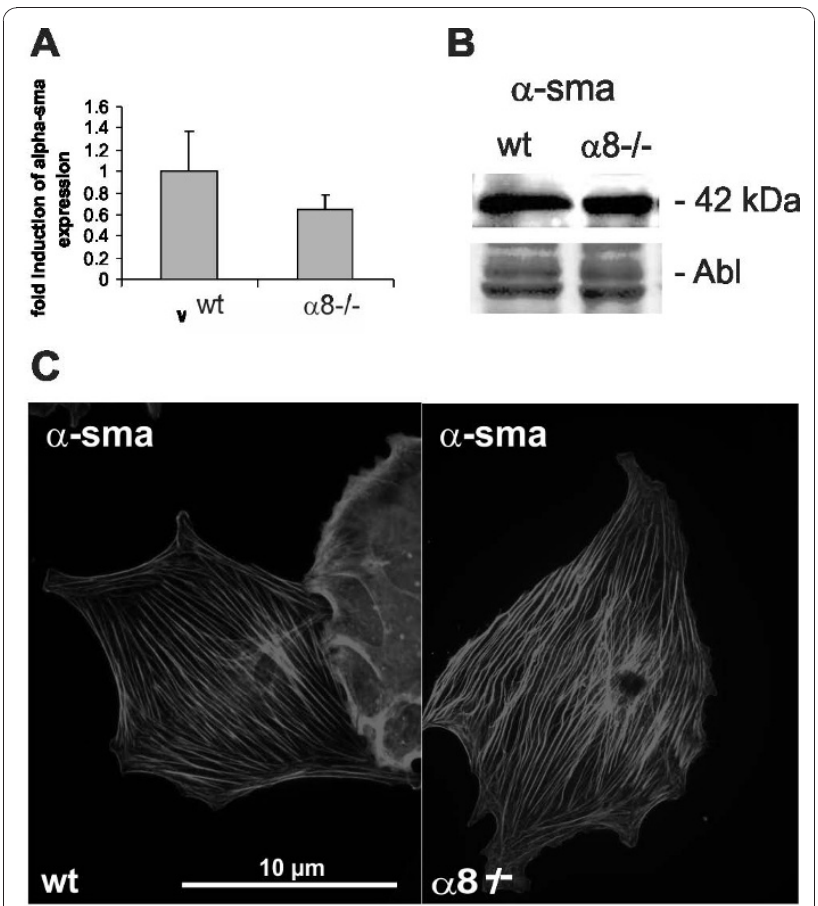

Figure 6 Comparison of $\alpha$-smooth muscle actin expression in wild type (wt) and $\alpha 8$ integrin-deficient ( $\alpha 8-/-)$ freshly isolated vascular smooth muscle cells. $\alpha 8$ integrin expression by real-time RT-PCR analysis (A), Western blot analysis with amido black (Abl) staining as loading control. (B) and immunofluorescent detection (C) (x1000). Results are representative for at least 3 similar experiments.

stress fibers with an inhibitor of rho family proteins resulted in inhibition of CTGF expression [32]. We could show that MCs lacking a8 integrin rearrange their actin cytoskeleton and downregulate CTGF.

Changes in the cytoskeletal architecture can alter cell adhesion and motility [33]. In a previous study, we showed that compared to wild type MCs, a8 integrin-

\section{CTGF}

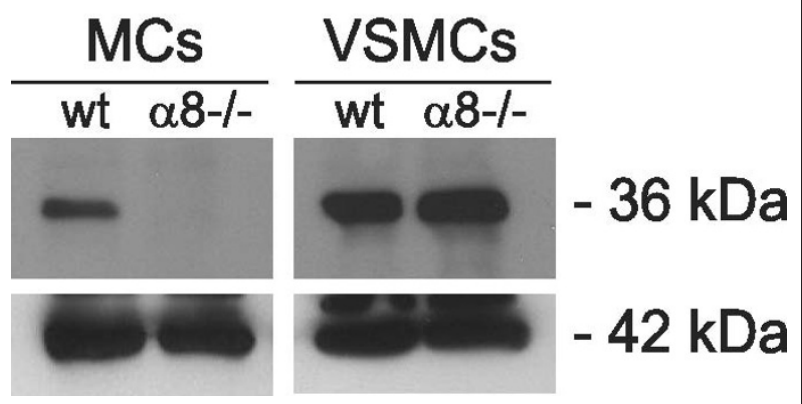

Figure 7 Connective tissue growth factor (CTGF; $36 \mathrm{kDa}$ ) protein expression in wild type (wt) and $\alpha 8$ integrin-deficient ( $\alpha 8-/-)$ mesangial cells (MCs) and vascular smooth muscle cells (VSCMs). Staining for $\beta$-actin was used as a loading control. Results are representative for 3 similar experiments. deficient MCs adhered weaker to fibronectin and vitronectin, two ligands for a8 integrin, but adhered more easily on collagens, which are not ligands for a8 integrin [18]. On the other hand, a8 integrin-deficient MCs migrated more easily on fibronectin or vitronectin than wild type cells [18]. These results support the notion that $\alpha 8$ integrin could serve as an anti-migratory integrin, keeping MCs resting at their native location. Firm adhesion, as mediated by $\alpha 8$ integrin, inhibits migration in many cell types [34]. Thus the decreased ability of $\alpha 8$ integrin-deficient MCs to adhere to fibronectin or vitronectin could contribute to the increased ability of these cells to migrate. Given these differences in migratory abilities, we hypothesized in the present study that wild type and $\alpha 8$ integrin-deficient MCs also differ in their cytoskeletal architecture and general morphology.

Downregulation of a-smooth muscle actin expression in a8 integrin-deficient MCs leads to a reduction in asmooth muscle actin containing stress fibers and consequently to a reduction in firm adhesion. This in turn seems to lead to increased cell motility of a8 integrindeficient MCs. Similar observations were made in VSMCs after siRNA knockdown of a8 integrin expression [20]: Treatment with a8 integrin siRNA reduced expression of a-smooth muscle actin and increased cell migration, which is in contrast to our findings in a8 integrin-deficient VSMCs, where both the a8 integrindeficient and the wild type genotype expressed a-smooth muscle actin in comparable amounts. The reasons for the discrepancy of the results of the studies in VSMCs after blockade of a8 integrin expression with siRNA and in a8 integrin-deficient VSMCs are unclear at present. Our results regarding a-smooth muscle actin expression in a8 integrin-deficient MCs are reminiscent of the findings of Zaghram et al. [20] after siRNA blockade of a8 integrin in VSMCs. We therefore wanted to investigate the differences in a8 integrin-deficient MCs and a8 integrin-deficient VSMCs: A compensatory increase of integrin chains a1, a2 and a6 was detected in a8 integrin-deficient MCs. A similar increase of integrin expression was not found in a8 integrin-deficient VSMCs. Thus, it seems possible that changes in the cytoskeletal architecture and a-smooth muscle actin expression in a8 integrin-deficient MCs is not a direct consequence of the lack of a8 integrin, but more likely due to the induction of other integrin chains. a6 integrin is usually not expressed in MCs, but is an integrin characteristic of epithelial cells, while a8 integrin is a typical mesenchymal integrin $[35,36]$. During kidney development, downregulation of a8 integrin, possibly by WT-1 [22], results in epithelialization of mesenchymal cells and in the formation of tubular structures [37]. For this reason, we tested if a8 integrin-deficient MCs exhibit reduced expression of other mesenchymal markers or increased 

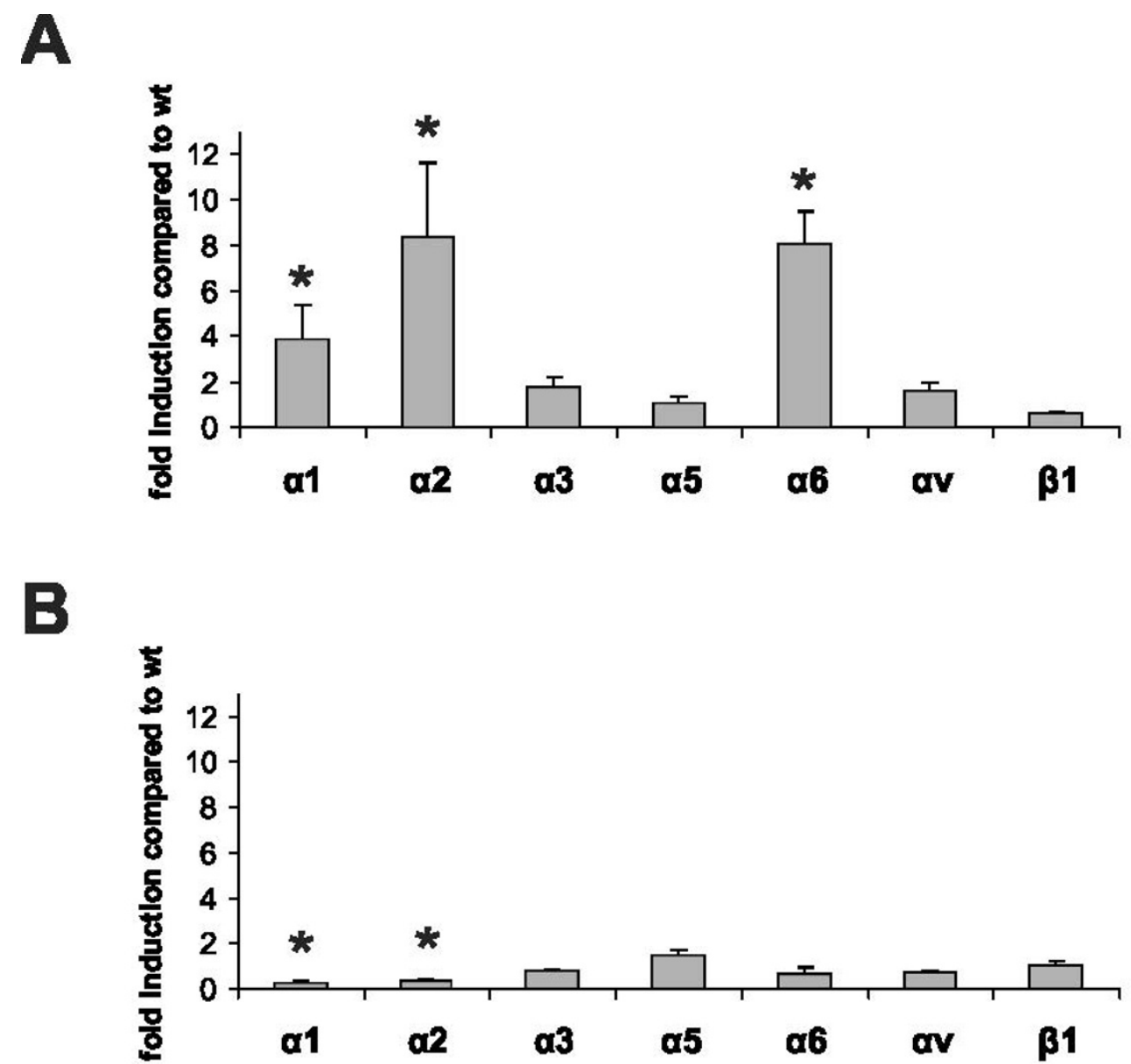

Figure 8 Real-time RT-PCR analysis of integrin chain $\alpha 1, \alpha 2, \alpha 3, \alpha 5, \alpha 6, \alpha v$ and $\beta 1$ expression profiles in wild type (wt) and $\alpha 8$ integrin-deficient ( $\alpha \mathbf{8}-/-)$ mesangial cells $(A)$ and freshly isolated vascular smooth muscle cells $(B)$. Results are representative for at least 3 similar experiments. Data are means \pm sd. ${ }^{*} p<0.05$ vs. wt.

expression of a typical epithelial marker, widely used in the detection of epithelial-mesenchymal transition $[38,39]$. A reduction of desmin expression was readily detected, but vimentin expression was not reduced and E-cadherin expression was very low in a8 integrindeficient MCs. These findings argue against the hypothesis that lack of a8 integrin, along with increased expression of $\mathrm{a} 1, \mathrm{a} 2$ and $\mathrm{a} 6$ integrins, leads to an epithelialization of MCs, but more likely might result in dedifferentiation of MCs. Why a8 integrin-deficient MCs undergo these changes in integrin expression and cytoskeletal architecture, while a8 integrin-deficient VSMCs do not, remains unclear. Discrepancies in the differentiation status might influence the ability of cells to dedifferentiate more easily than others. MCs and VSMCs might also use distinct transcriptional mechanisms, like it was described for smooth muscle cell and myofibroblast a-smooth muscle actin expression [40]. Moreover, no explanation exists to date as to why VSMCs after blockade of a8 integrin with siRNA behave differently from a8 integrin-deficient VSMCs regarding a-smooth muscle actin expression and cytoskeletal rearrangements. As shown by Zargham et al. [20], blockade of a8 integrin with siRNA results in a dysregulation of the expression of other integrins, like an increased expression of the a2, a5 and av chains, or reduced expression of the a1 chain. In our isolations of a8 integrindeficient VSMCs we did not observe significant increases in the expression of the a2, a 5 and av chains, while the expression of the a1 chain indeed was reduced. One has to be aware that acute blockade of a8 integrin with siRNA in VSMCs might not be consistent with a genetic knockdown of a8, which is more comparable to a chronic deficiency from the time of VSMC differentiation on. As a consequence, many regulatory pathways might differ in the two cell types. Moreover, the findings with blockade of a8 integrin with siRNA was obtained in rat 


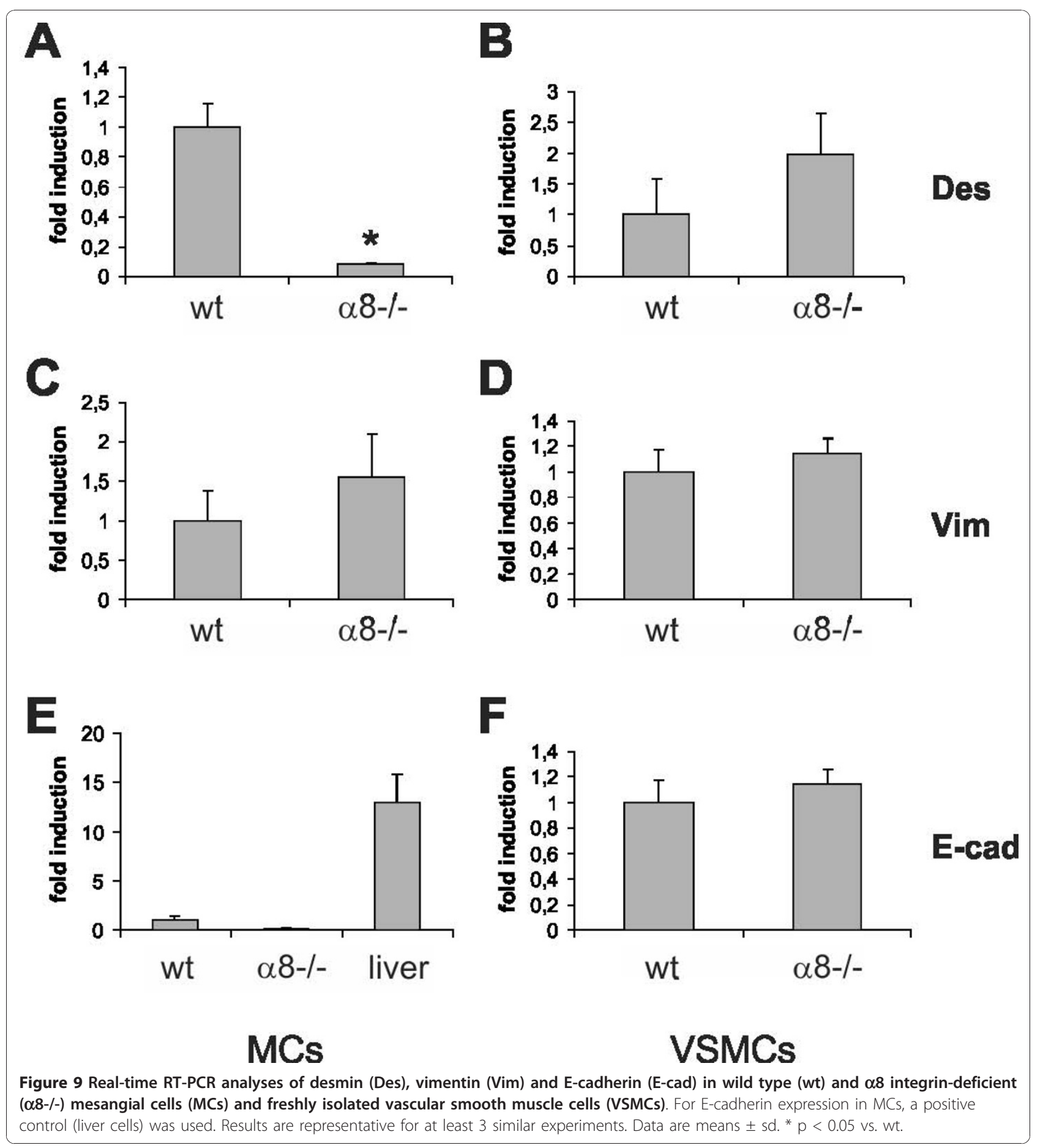

VSMCs [20], while our data are derived from mouse VSMCs. Species differences might exist with regard to VSMC biology.

Finally, differences in the properties of MCs and VSMCc lacking a8 integrin were detected regarding cell growth. While a8 integrin-deficient MCs had significantly increased proliferation rates on ligands for a8 integrin compared to wild type MCs [18], wild type and $\alpha 8$ integrin-deficient VSMCs showed a comparable growth response after stimulation. Thus it is conceivable that the cytoskeletal and matrix receptor changes in $\alpha 8$ integrindeficient MCs may result in changes in proliferative capacities of these cells. Both $\alpha 2$ and $\alpha 6$ integrin chains, which are upregulated in $\alpha 8$ integrin-deficient MCs, can promote cell proliferation $[41,42]$. On the other hand, increased proliferation rates in MCs lacking a8 integrin 


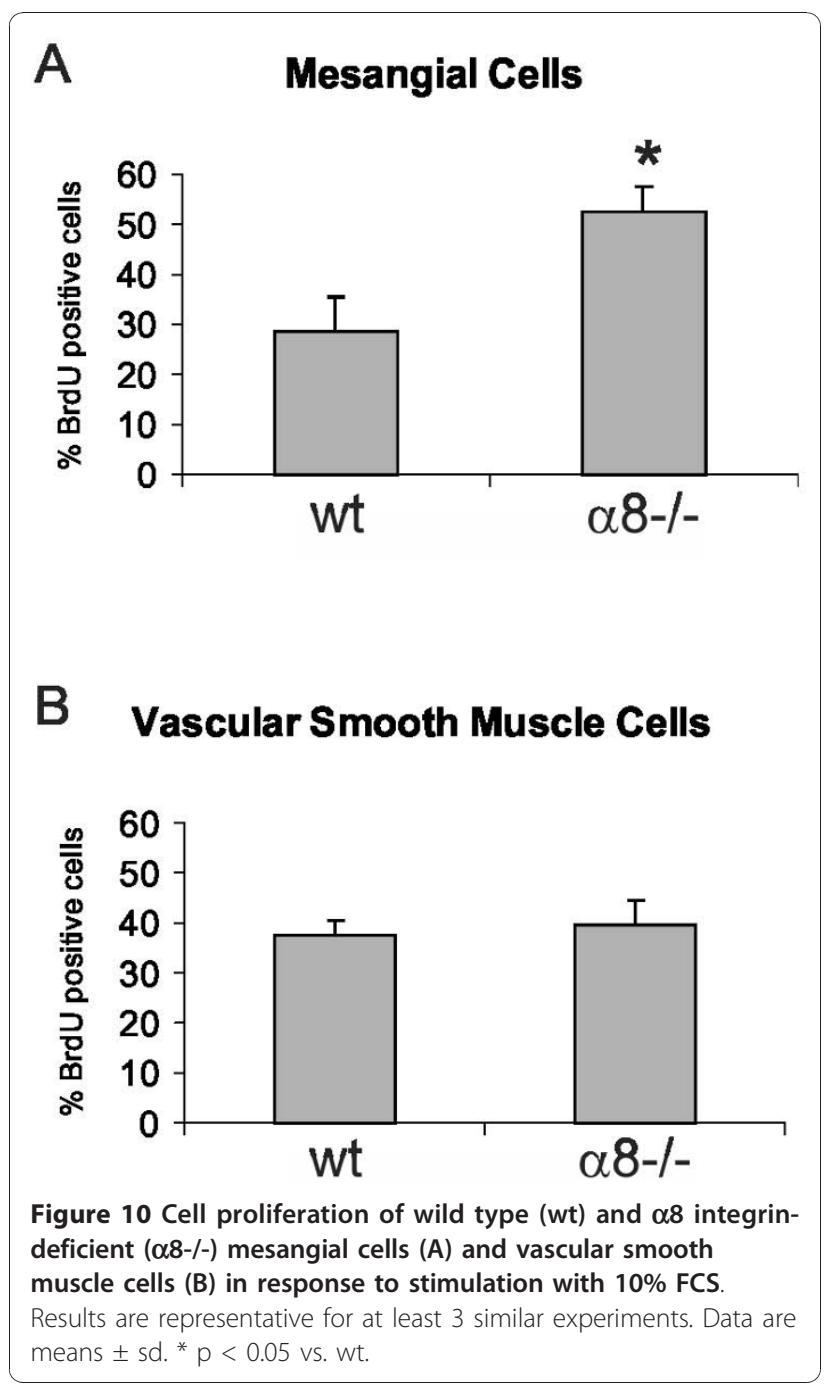

might be a consequence of rho-mediated disruption of actin stress fibers, leading to increased expression of CTGF, which was described to act anti-proliferative [31].

\section{Conclusion}

A lack of a8 integrin appears to be of little consequence in VSMCs whereas the deficiency of this integrin has profound effects on the MC phenotype. The different abilities of both cell types to induce other integrin chains might well result in different phosphorylation patterns of kinases involved in integrin signaling, which could lead to a different activation of signaling cascades, causing differences in cytoskeletal characteristics and proliferation capacities.

\section{Methods}

\section{Cultivation of mouse mesangial cells and vascular} smooth muscle cells

Cells used in this study were obtained from organs of wild type or $\alpha 8$ integrin-deficient mice (obtained from $\mathrm{U}$.
Müller, Basel). Animal caretaking was performed according to the guidelines of the American Physiological Society and approved by local government authorities. Mesangial cells (MCs) were isolated from kidneys by the sieving method [43] using 63, 75 and $38 \mu \mathrm{m}$ grid sieves. Cultured wild type and $\alpha 8$ integrin-deficient MCs were characterized as described [18]. MCs were grown in Dulbecco's modified Eagle's Medium (DMEM; PAA Laboratories $\mathrm{GmbH}$, Linz, Austria) containing 10\% FCS, 5 нg/ $\mathrm{ml}$ insulin, $5 \mu \mathrm{g} / \mathrm{ml}$ plasmocin (TEBU, Frankfurt, Germany) and $2 \mathrm{mM}$ L-glutamine (Sigma, Deisenhofen, Germany) in a $95 \%$ air - $5 \% \mathrm{CO}_{2}$ humified atmosphere at $37^{\circ}$ C. MCs were used for experiments in passages 5-10.

Vascular smooth muscle cells (VSMCs) were isolated from mouse aorta similar as described by Strehlow et al. [44] for rat vascular smooth muscle cells. Briefly, the aortas were excised, washed in phosphate-buffered saline with $1 \%$ penicillin-streptomycin and fat was removed with a fine forceps. The aortas were then incubated in DMEM containing $1 \mathrm{mg} / \mathrm{ml}$ collagenase type I (Sigma), $0.3 \mathrm{mg} / \mathrm{ml}$ elastase (Serva, Heidelberg, Germany) and $0.3 \mathrm{mg} / \mathrm{ml}$ trypsin inhibitor type II (Sigma) for 15 to 20 minutes at $37^{\circ} \mathrm{C}$. The aorta was washed and the adventitia was stripped with fine forceps. The vessels were incised longitudinally and the endothelial cells were gently scraped off. The aortas were then minced with scissors and transferred to reaction tubes containing the same enzymatic solution as described above, incubated in $37^{\circ} \mathrm{C}$ for 60 to 90 minutes until $90 \%$ of the cells were dispersed under the microscope. The cells were centrifuged at $5000 \mathrm{rpm}$ for $2 \mathrm{~min}$ utes, then resuspended in $3 \mathrm{ml}$ DMEM with $20 \%$ fetal calf serum (FCS), 2\% penicillin-streptomycin, and cultured in plates or flasks in a $95 \%$ air - $5 \% \mathrm{CO}_{2}$ humidified atmosphere at $37^{\circ} \mathrm{C}$ for experiments. Cultured cells were verified to be VSMCs by immunostaining with anti-smooth muscle actin antibody (Sigma).

For detection of cytoskeletal components, MCs and VSMCs were allowed to attach on culture slides coated with $10 \mu \mathrm{g} / \mathrm{ml}$ fibronectin for 24 hours.

\section{Isolation of mRNA and Real-time PCR}

To evaluate mRNA expression levels, total RNA was obtained from harvested cells by extraction with RNeasy ${ }^{\odot}$ Mini columns (Quiagen, Hilden, Germany). First-strand cDNA was synthesized with TaqMan reverse transcription reagents (Applied Biosystems, Weiterstadt, Germany) using random hexamers as primers. Final RNA concentration in the reaction mixture was adjusted to 0.1 ng/ $\mu \mathrm{L}$. Reactions without Multiscribe reverse transcriptase were used as negative controls for genomic DNA contamination. PCR was performed with an ABI PRISM 7000 Sequence Detector System and SYBR Green or TaqMan reagents (Applied Biosystems) according to the manufacturer's instructions. The relative amount 
of the specific mRNA was normalized with respect to $18 \mathrm{~S}$ rRNA. Primers used for amplification are listed in Table 1. For detection of E-cadherin mRNA, a TaqMan probe was used: 5'-GTC ACA GAC CCC ACG ACC AAT GAT-3'. All samples were run in triplicates.

\section{Western Blot Analysis}

Protein concentration of cell lysates was determined using a protein assay kit (Pierce, Rockford, IL). Protein samples containing $30 \mu \mathrm{g}$ total protein were denatured by boiling for five minutes and separated on a $8 \%$ denaturing SDSPAGE gel. After electrophoresis, the gels were electroblotted onto PVDF membranes (Pall Filtron, Karlstein, Germany), blocked with 5\% horse serum/TBS/0.1\% Tween 20 for 2 hours and incubated with the primary antibody overnight. Immunoreactivity was visualized with a secondary horseradish peroxidase-conjugated anti-rabbit IgG antibody or anti-mouse IgG antibody (both from Santa Cruz Biotechnology, Heidelberg, Germany), using the ECL system according to the manufacturer's instructions (Amersham, Braunschweig, Germany).

\section{Immunocytochemistry}

MCs and VSMCs were seeded on glass 8-well chamber slides blocked with $2 \%$ BSA. Cells were allowed to adhere for $24 \mathrm{~h}$. Then, supernates were removed, adherent cells were rinsed $3 \times$ with PBS and fixed in 3\% paraformaldehyde for $20 \mathrm{~min}$. After blockade of free aldehyde groups with 50 $\mathrm{mM}$ ammonium chloride, cells were permeabilized by $1 \%$ Triton X-100 and nonspecific binding was blocked using $100 \%$ FCS. Cells were incubated with the primary antibodies overnight, followed by a CY3-labelled goat anti-rabbit or anti-mouse immunoglobulin G (Dianova) as secondary antibody and embedding in Tris-buffered Mowiol, $\mathrm{pH}$ 8,6 (Hoechst). F-actin was visualized with phalloidin from Molecular Probes (Leiden, The Netherlands).

\section{Antibodies}

The rabbit polyclonal antiserum to $\alpha 8$ integrin was kindly provided by Dr. Ulrich Muller, San Diego and used at a dilution of 1:200 as described before [43]. A polyclonal antibody to CTGF (Santa Cruz Biotechnology) was used in a dilution of 1:1000. A monoclonal antibody to smooth muscle actin (DAKO Diagnostika, Hamburg, Germany) was used at a dilution of 1:50 for immunocytochemistry or 1:1000 for Western blot analysis. A polyclonal antibody to vinculin (Santa Cruz Biotechnology) was used in a dilution of 1:500 for immunocytochemistry.

\section{Determination of cell proliferation}

To assess cell growth, a 5-bromo-2'-deoxy-uridine (BrdU) incorporation assay into cellular DNA was performed using a BrdU labeling and detection kit (\#1299964; Roche Mannheim, Germany). Cells were washed two times with PBS and serum-starved for 72 hours in medium containing $0.1 \%$ FCS. After trypsinating and washing they were seeded into culture slides (Falcon, HTS; Becton Dickinson, Heidelberg, Germany) which had been coated with $10 \mu \mathrm{g} / \mathrm{ml}$ fibronectin and blocked with $2 \%$ BSA. After a 12 -hour resting period allowing the cells to attach to the matrix, they were incubated with medium containing 10\% FCS for 48 hours. For the last two hours of incubation, BrdU was added. Cells were then fixed with $70 \%$ ethanol (in 50 $\mu \mathrm{M}$ glycine buffer; $\mathrm{pH} 2.0$ ) and processed following the manufacturer's instructions. Incorporated BrdU was detected by an alkaline phosphatase-conjugated secondary antibody reacting with an NBT/X-phosphate substrate. Cells were counterstained with hematoxylin. Nuclei with a positive staining for BrdU were counted. Results shown are representative for at least three independent experiments.

Table 1 Primer pairs for Sybr green analysis

\begin{tabular}{|c|c|c|}
\hline & forward & reverse \\
\hline$\alpha$-smooth muscle actin & 5'-CCC TGA AGA GCA TCC GAC AC-3' & 5'-GCC TTA GGG TTC AGT GGT GC-3' \\
\hline$\alpha 1$ integrin & 5'-CCA GTC AGC AGC TTC GTT TGA-3' & 5'-TC CAG TCA TAG GCT CCC ACA G-3' \\
\hline$\alpha 2$ integrin & 5'-TGA CCA GGT TCT GCA GGA TAG A-3' & 5'-AGT AGA AAT TGC AGC CAC AGA GTA AC-3' \\
\hline$\alpha 3$ integrin & 5'-AGG CAC AGG CTA TGG AGA ATC A-3' & 5'-CGC ACT CTT TCT GGA AGT GGA C-3' \\
\hline$\alpha 5$ integrin & 5'-TCG GAG CAA CAG TTC GGG-3' & 5'-GTG GAG CAC ATG CCA AGA TG-3' \\
\hline$\alpha 6$ integrin & 5'-TCC CCG ACT GGC ATA ATT ACC-3' & 5'-CGA TGT CCC CTC GAG AAC C-3' \\
\hline$\alpha 8$ integrin & 5'-TCA AGG CGA GGA ACA GCA A-3' & 5'-CCT TGG GAA CCC GAT GGT-3' \\
\hline$\alpha \vee$ integrin & 5'-GGA GCT TाT GGT GTG GAT CG-3' & 5'-GAC AAC GGG TCT GGC TCT GTA-3' \\
\hline$\beta 1$ integrin & 5'-TGG CAA CAA TGA AGC TAT CGT G-3' & 5'-GTA GGA CAG TCT GGA GTC TCC ACA-3' \\
\hline desmin & 5'-GTG AAG ATG GCC TTG GAT GT-3' & $5^{\prime}-T T G$ AGA GCA GAG AAG GTC TGG-3' \\
\hline vimentin & 5'-ACG ATC TCA CCC TCA GGG CT-3' & 5'-GGG TCG CTG AGT CAG TGG AT-3' \\
\hline e-cadherin & 5'-AAG TGA CCG ATG ATG ATG CC-3' & 5'-CTT CAT TCA CGT CTA CCA CGT-3' \\
\hline $18 \mathrm{~S}$ & 5'-TTG ATT AAG TCC CTG CCC TाT GT-3' & 5'-CGA TCC GAG GGC CTC ACT A-3' \\
\hline
\end{tabular}




\section{Statistical analyses}

A t-test was used to test significance of differences between groups. A P-value $<0.05$ was considered significant. The procedures were carried out using SPSS software (SPSS Inc., Chicago, USA). Values are displayed as means \pm standard deviation $(\mathrm{SD})$.

\section{List of Abbreviations}

CTGF: connective tissue growth factor; MCs: mesangial cells; VSMCs: vascular smooth muscle cells

\section{Acknowledgements}

We thank Dr. Ulrich Muller, San Diego for kindly providing us with $\alpha 8$ integrin-deficient mice and Dr. Kerstin Strehlow, Homburg for assistance in developing the technique of mouse vascular smooth muscle cell isolation. This study was supported by a grant from the Deutsche

Forschungsgemeinschaft, Bonn; Sonderforschungsbereich 423, TP A2

\section{Author details}

${ }^{1}$ Hospital for Children and Adolescents, Universität Erlangen-Nürnberg, Loschgestrasse 15, 91054 Erlangen, Germany. 'Department of Nephrology and Hypertension, Universität Erlangen-Nürnberg, Loschgestrasse 8, 91054 Erlangen, Germany.

\section{Authors' contributions}

$I M, G V, A J$ and ZO carried out the cell culture experiments, CZ and FF analysed the data and helped drafting the manuscript, KFH performed the statistical analyses, MGS, WR and AH participated in the design and coordination of the study, AH drafted the manuscript. All authors have read and approved the final manuscript.

Received: 21 June 2010 Accepted: 31 December 2010

Published: 31 December 2010

\section{References}

1. Danen $E H$, Sonnenberg A: Integrins in regulation of tissue development and function. J Pathol 2003, 201(4):632-641.

2. Giancotti FG: Complexity and specificity of integrin signalling. Nat Cell Biol 2000, 2(1):E13-14.

3. Wiesner S, Legate KR, Fassler R: Integrin-actin interactions. Cell Mol Life Sci 2005, 62(10):1081-1099.

4. Hynes RO: Integrins: bidirectional, allosteric signaling machines. Cell 2002, 110(6):673-687.

5. Delon I, Brown NH: Integrins and the actin cytoskeleton. Curr Opin Cell Biol 2007, 19(1):43-50.

6. Chen CA, Hwang JC, Guh JY, Tsai JC, Chen HC: TGF-beta1 and integrin synergistically facilitate the differentiation of rat podocytes by increasing alpha-smooth muscle actin expression. Trans/ Res 2006, 148(3):134-141.

7. Blattner SM, Kretzler M: Integrin-linked kinase in renal disease: connecting cell-matrix interaction to the cytoskeleton. Curr Opin Nephrol Hypertens 2005, 14(4):404-410.

8. Graness A, Giehl K, Goppelt-Struebe M: Differential involvement of the integrin-linked kinase (ILK) in RhoA-dependent rearrangement of F-actin fibers and induction of connective tissue growth factor (CTGF). Cell Signal 2006, 18(4):433-440.

9. White LR, Blanchette JB, Ren L, Awn A, Trpkov K, Muruve DA: The characterization of alpha5-integrin expression on tubular epithelium during renal injury. Am J Physiol Renal Physiol 2007, 292(2):F567-576.

10. Lakhe-Reddy S, Khan S, Konieczkowski M, Jarad G, Wu KL, Reichardt LF, Takai Y, Bruggeman LA, Wang B, Sedor JR, et al: Beta8 integrin binds Rho GDP dissociation inhibitor-1 and activates Rac1 to inhibit mesangial cell myofibroblast differentiation. J Biol Chem 2006, 281(28):19688-19699.

11. Lygoe KA, Norman JT, Marshall JF, Lewis MP: AlphaV integrins play an important role in myofibroblast differentiation. Wound Repair Regen 2004, 12(4):461-470.

12. Li Y, Yang J, Dai C, Wu C, Liu Y: Role for integrin-linked kinase in mediating tubular epithelial to mesenchymal transition and renal interstitial fibrogenesis. J Clin Invest 2003, 112(4):503-516.
13. Shimizu M, Kondo S, Urushihara M, Takamatsu M, Kanemoto K, Nagata M, Kagami S: Role of integrin-linked kinase in epithelial-mesenchymal transition in crescent formation of experimental glomerulonephritis. Nephrol Dial Transplant 2006, 21(9):2380-2390.

14. Muller U, Bossy B, Venstrom K, Reichardt LF: Integrin alpha 8 beta 1 promotes attachment, cell spreading, and neurite outgrowth on fibronectin. Mol Biol Cell 1995, 6(4):433-448.

15. Denda S, Muller U, Crossin KL, Erickson HP, Reichardt LF: Utilization of a soluble integrin-alkaline phosphatase chimera to characterize integrin alpha 8 beta 1 receptor interactions with tenascin: murine alpha 8 beta 1 binds to the RGD site in tenascin- $C$ fragments, but not to native tenascin-C. Biochemistry 1998, 37(16):5464-5474.

16. Denda S, Reichardt LF, Muller U: Identification of osteopontin as a novel ligand for the integrin alpha 8 beta 1 and potential roles for this integrinligand interaction in kidney morphogenesis. Mol Biol Cell 1998, 9(6):1425-1435.

17. Brandenberger R, Schmidt A, Linton J, Wang D, Backus C, Denda S, Muller $U$, Reichardt LF: Identification and characterization of a novel extracellular matrix protein nephronectin that is associated with integrin alpha8beta1 in the embryonic kidney. J Cell Biol 2001, 154(2):447-458.

18. Bieritz B, Spessotto P, Colombatti A, Jahn A, Prols F, Hartner A: Role of alpha8 integrin in mesangial cell adhesion, migration, and proliferation. Kidney Int 2003, 64(1):119-127.

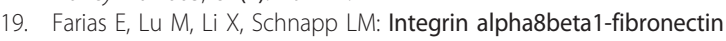
interactions promote cell survival via PI3 kinase pathway. Biochem Biophys Res Commun 2005, 329(1):305-311.

20. Zargham $R$, Thibault $G$ : alpha8 Integrin expression is required for maintenance of the smooth muscle cell differentiated phenotype. Cardiovasc Res 2006, 71(1):170-178.

21. Zargham R, Touyz RM, Thibault G: alpha8 Integrin overexpression in dedifferentiated vascular smooth muscle cells attenuates migratory activity and restores the characteristics of the differentiated phenotype. Atherosclerosis 2007, 195(2):303-312.

22. Hosono S, Luo X, Hyink DP, Schnapp LM, Wilson PD, Burrow CR, Reddy JC, Atweh GF, Licht JD: WT1 expression induces features of renal epithelial differentiation in mesenchymal fibroblasts. Oncogene 1999, 18(2):417-427.

23. Zargham R, Thibault G: alpha8beta1 Integrin expression in the rat carotid artery: involvement in smooth muscle cell migration and neointima formation. Cardiovasc Res 2005, 65(4):813-822.

24. Levine D, Rockey DC, Milner TA, Breuss JM, Fallon JT, Schnapp LM: Expression of the integrin alpha8beta1 during pulmonary and hepatic fibrosis. Am J Pathol 2000, 156(6):1927-1935

25. Hartner A, Cordasic N, Klanke B, Muller U, Sterzel RB, Hilgers KF: The alpha8 integrin chain affords mechanical stability to the glomerular capillary tuft in hypertensive glomerular disease. Am J Pathol 2002, 160(3):861-867.

26. Haas CS, Amann K, Schittny J, Blaser B, Muller U, Hartner A: Glomerular and renal vascular structural changes in alpha8 integrin-deficient mice. J Am Soc Nephrol 2003, 14(9):2288-2296.

27. Hintermann E, Yang N, O'Sullivan D, Higgins JM, Quaranta V: Integrin alpha6beta4-erbB2 complex inhibits haptotaxis by up-regulating Ecadherin cell-cell junctions in keratinocytes. J Biol Chem 2005, 280(9):8004-8015.

28. DeMali KA, Wennerberg K, Burridge K: Integrin signaling to the actin cytoskeleton. Curr Opin Cell Biol 2003, 15(5):572-582.

29. Wu C, Dedhar S: Integrin-linked kinase (ILK) and its interactors: a new paradigm for the coupling of extracellular matrix to actin cytoskeleton and signaling complexes. J Cell Biol 2001, 155(4):505-510.

30. Samarakoon R, Goppelt-Struebe M, Higgins PJ: Linking cell structure to gene regulation: signaling events and expression controls on the model genes PAl-1 and CTGF. Cell Signal 2010, 22(10):1413-1419.

31. Hishikawa K, Oemar BS, Tanner FC, Nakaki T, Fujii T, Luscher TF: Overexpression of connective tissue growth factor gene induces apoptosis in human aortic smooth muscle cells. Circulation 1999, 100(20):2108-2112

32. Hahn A, Heusinger-Ribeiro J, Lanz T, Zenkel S, Goppelt-Struebe M: Induction of connective tissue growth factor by activation of heptahelical receptors. Modulation by Rho proteins and the actin cytoskeleton. J Biol Chem 2000, 275(48):37429-37435.

33. Lo SH: Focal adhesions: what's new inside. Dev Biol 2006, 294(2):280-291.

34. Holly SP, Larson MK, Parise LV: Multiple roles of integrins in cell motility. Exp Cell Res 2000, 261(1):69-74. 
35. Schnapp LM, Breuss JM, Ramos DM, Sheppard D, Pytela R: Sequence and tissue distribution of the human integrin alpha 8 subunit: a beta 1associated alpha subunit expressed in smooth muscle cells. J Cell Sci 1995, 108(Pt 2):537-544.

36. Hay ED, Zuk A: Transformations between epithelium and mesenchyme: normal, pathological, and experimentally induced. Am J Kidney Dis 1995 26(4):678-690.

37. Muller U, Brandli AW: Cell adhesion molecules and extracellular-matrix constituents in kidney development and disease. J Cell Sci 1999, 112(Pt 22):3855-3867.

38. Strutz F, Zeisberg M, Ziyadeh FN, Yang CQ, Kalluri R, Muller GA, Neilson EG: Role of basic fibroblast growth factor-2 in epithelial-mesenchymal transformation. Kidney Int 2002, 61(5):1714-1728.

39. Vongwiwatana A, Tasanarong A, Rayner DC, Melk A, Halloran PF: Epithelial to mesenchymal transition during late deterioration of human kidney transplants: the role of tubular cells in fibrogenesis. Am J Transplant 2005, 5(6):1367-1374.

40. Gan Q, Yoshida T, Li J, Owens GK: Smooth muscle cells and myofibroblasts use distinct transcriptional mechanisms for smooth muscle alpha-actin expression. Circ Res 2007, 101(9):883-892.

41. Grzesiak JJ, Bouvet M: Activation of the alpha2beta1 integrin-mediated malignant phenotype on type I collagen in pancreatic cancer cells by shifts in the concentrations of extracellular $\mathrm{Mg} 2+$ and $\mathrm{Ca} 2+$. Int J Cancer 2008, 122(10):2199-2209.

42. Fang IM, Yang $\mathrm{CH}$, Yang $\mathrm{CM}$, Chen MS: Overexpression of integrin alpha6 and beta4 enhances adhesion and proliferation of human retinal pigment epithelial cells on layers of porcine Bruch's membrane. Exp Eye Res 2009, 88(1):12-21.

43. Hartner A, Schocklmann H, Prols F, Muller U, Sterzel RB: Alpha8 integrin in glomerular mesangial cells and in experimental glomerulonephritis. Kidney Int 1999, 56(4):1468-1480.

44. Strehlow K, Rotter S, Wassmann S, Adam O, Grohe C, Laufs K, Bohm M, Nickenig G: Modulation of antioxidant enzyme expression and function by estrogen. Circ Res 2003, 93(2):170-177.

doi:10.1186/1471-2121-11-102

Cite this article as: Marek et al: Lack of $\alpha 8$ integrin leads to morphological changes in renal mesangial cells, but not in vascular smooth muscle cells. BMC Cell Biology 2010 11:102.

\section{Submit your next manuscript to BioMed Central} and take full advantage of:

- Convenient online submission

- Thorough peer review

- No space constraints or color figure charges

- Immediate publication on acceptance

- Inclusion in PubMed, CAS, Scopus and Google Scholar

- Research which is freely available for redistribution

Submit your manuscript at www.biomedcentral.com/submit
Ciomed Central 